\title{
Cutaneous and bowel vascular malformation
}

\section{Pankaj Kumar Garg}

Published online: 4 February 2015

(C) Indian Society of Gastroenterology 2015

The blue rubber bleb nevus syndrome (BRBNS) is a rare disorder characterized by vascular malformations of the skin and gastrointestinal (GI) tract. The term was coined by William Bean (so also known as Bean syndrome) in 1958; though an association between cavernous hemangiomas of the skin and similar lesions in the GI tract was first described by Gascoyen in 1860 [1]. The skin lesions are characterized by the presence of blue or blackish-blue, compressible, nontender nodules of varying sizes ( $5 \mathrm{~mm}$ to a few centimeters), mainly present over the trunk, soles, and palms but may be

Fig. 1 a Clinical photograph of the patient shows multiple, sessile, bluish, smooth-surfaced nodules in the neck and $\mathbf{b}$ colonoscopic image shows bluish nevus in the sigmoid colon
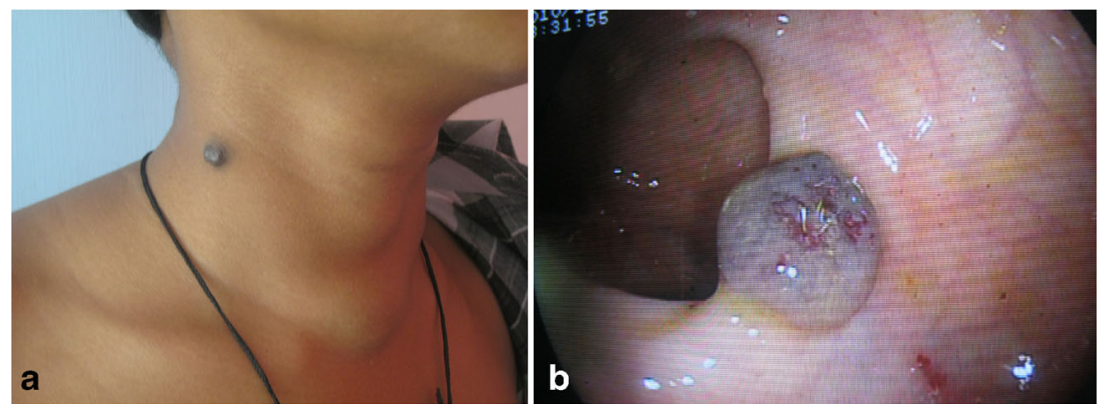

seen anywhere in the skin. The skin lesions are mostly asymptomatic. In BRBNS, vascular lesions in the GI tract can be numerous and diffuse and can be located anywhere between the oral and anal mucosa. This image is from a 16-year-old boy who presented with multiple sessile, bluish, smooth-surfaced nodules distributed over the face, neck, back, and all four limbs, ranging in size from 1 to $3 \mathrm{~cm}$ (Fig. 1a). These were non-tender, soft, rubbery, and compressible. Colonoscopy revealed the characteristic multiple bluish nevi in the rectum and sigmoid colon (Fig. 1b).

\section{Reference}

1. Suma GN, Ravi Prakash SM, Rao D, Goel S. Blue rubber bleb nevus syndrome: prominent oral findings. Indian J Dermatol Venereol Leprol. 2010;76:168-71.
P. K. Garg $(\bowtie)$

Department of Surgery, University College of Medical Sciences and

Guru Teg Bahadur Hospital, University of Delhi, Dilshad Garden,

New Delhi 110 095, India

e-mail: dr.pankajgarg@gmail.com 